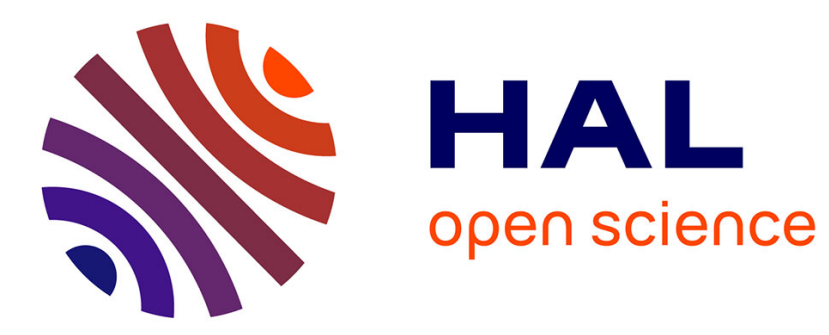

\title{
Generation of coherent acoustic beams in solids by mixing of counterpropagating, detuned optical beams
}

\author{
Vincent Laude, Maria Korotyaeva, Jean-Charles Beugnot
}

\section{To cite this version:}

Vincent Laude, Maria Korotyaeva, Jean-Charles Beugnot. Generation of coherent acoustic beams in solids by mixing of counterpropagating, detuned optical beams. Applied optics, 2018, 57 (10), pp.C77-C82. 10.1364/AO.57.000C77 . hal-02131447

\section{HAL Id: hal-02131447 \\ https://hal.science/hal-02131447}

Submitted on 16 May 2019

HAL is a multi-disciplinary open access archive for the deposit and dissemination of scientific research documents, whether they are published or not. The documents may come from teaching and research institutions in France or abroad, or from public or private research centers.
L'archive ouverte pluridisciplinaire HAL, est destinée au dépôt et à la diffusion de documents scientifiques de niveau recherche, publiés ou non, émanant des établissements d'enseignement et de recherche français ou étrangers, des laboratoires publics ou privés. 


\title{
Generation of coherent acoustic beams in solids by mixing of counterpropagating, detuned optical beams [invited]
}

\author{
Vincent Laude ${ }^{1, *}$, Maria E. Korotyaeva ${ }^{1}$, And Jean-Charles Beugnot ${ }^{1}$ \\ ${ }^{1}$ Institut FEMTO-ST, CNRS, Univ. Bourgogne Franche-Comté, 15B avenue des Montboucons, 25030 Besançon, France \\ ${ }^{*}$ Corresponding author: vincent.laude@femto-st.fr
}

Compiled January 16, 2018

\begin{abstract}
We model the generation of coherent acoustic beams in a homogeneous solid from the interference of two oppositely propagating, detuned, optical laser beams. This configuration is reciprocal to Brillouin light scattering in the backward interaction arrangement. Generation of a confined ultrasound beam is predicted, close to the Brillouin frequency. Optoacoustic gain spectra and beam shapes are obtained numerically using a finite element model. The acoustic spectra are non-symmetrical, i.e. non-Lorentzian, and result from excitation of the continuum of bulk elastic waves. The acoustic beam width correspondingly varies with detuning frequency and optical beam waist. () 2018 Optical Society of America
\end{abstract}

OCIS codes: (290.5830) Scattering, Brillouin; (110.5125) Photoacoustics; (190.2055) Dynamic gratings.

http://dx.doi.org/10.1364/ao.XX.XXXXXX

\section{INTRODUCTION}

The mixing of two frequency detuned optical waves in a solid medium offers a means of generating coherent hypersound in the bulk [1]. Such an electrostriction process relies on the photoelastic effect $[2,3]$, whereby the square of the optical field induces a mechanical stress in the medium. When the stress distribution is phase matched with a particular elastic wave in the medium, i.e. when the interference wavelength and the detuning frequency satisfy the relevant dispersion relation, optoacoustic generation is in principle at a maximum.

Electrostriction is known to be intimately related to Brillouin light scattering (BLS) by acoustic phonons, of thermal origin, or elastic waves, for instance produced by a transducer. Actually, both effects can be described in a unified manner in energetic terms, using either a Lagrangian or a Hamiltonian for the coupled optical and elastic wave fields [4-6]. In Brillouin light scattering experiments, the backward scattering configuration is often used, as it allows to impose very precisely the acoustic wavevector direction and magnitude. BLS spectra can then be obtained by observing the backscattered light amplitude as a function of frequency. Shear and longitudinal phonons appear as Lorentzian peaks centered on the Brillouin frequency $\omega_{B}=2 n V / \lambda$, with $n$ the effective refractive index, $V$ the acoustic phase velocity, and $\lambda$ the vacuum wavelength of light. The factor 2 is typical of backscattering and the phonon wavenumber is twice the optical wavenumber in the medium, or $q=4 \pi n / \lambda$. The same backscattering configuration is used for BLS measure- ments in optical fibers and waveguides. In this case, $n$ is the effective index of a guided optical mode and $V$ is generally interpreted as the phase velocity of an elastic mode of the waveguide.

In this paper, we consider photoelastic generation of hypersound in the backward interaction arrangement. This arrangement has been considered in recent years in optical fibers and waveguides, and many fascinating observations have been made, including the existence of hybrid phonons in photonic crystal fibers [7], nanoscale optical waveguides [8-10], surface acoustic waves in microwires [11], or light storage in integrated photonic circuit waveguides [12,13]. The specificity of our work is that we consider optoacoustic generation in the bulk of homogeneous media, i.e. in the absence of any waveguide for the generated elastic waves. The absence of a waveguide implies the non existence of a basis of guided elastic modes, and hence the non applicability of descriptions of optoacoustic gain relying on overlap integrals [14, 15].

In the following, we propose a photoelastic generation model adapted to the case of bulk homogeneous media, in the case that the interaction length of the two counterpropagating optical Gaussian beams can be considered very long. We find that electrostriction leads to the generation of confined beams of elastic waves, centered on the optical stress distribution and whose lateral extension depends on the angular-spectrum contents of the optical beams and on the frequency detuning. Optoacoustic spectra are argued to be formed from the continuum of bulk elastic waves of the propagation medium. 


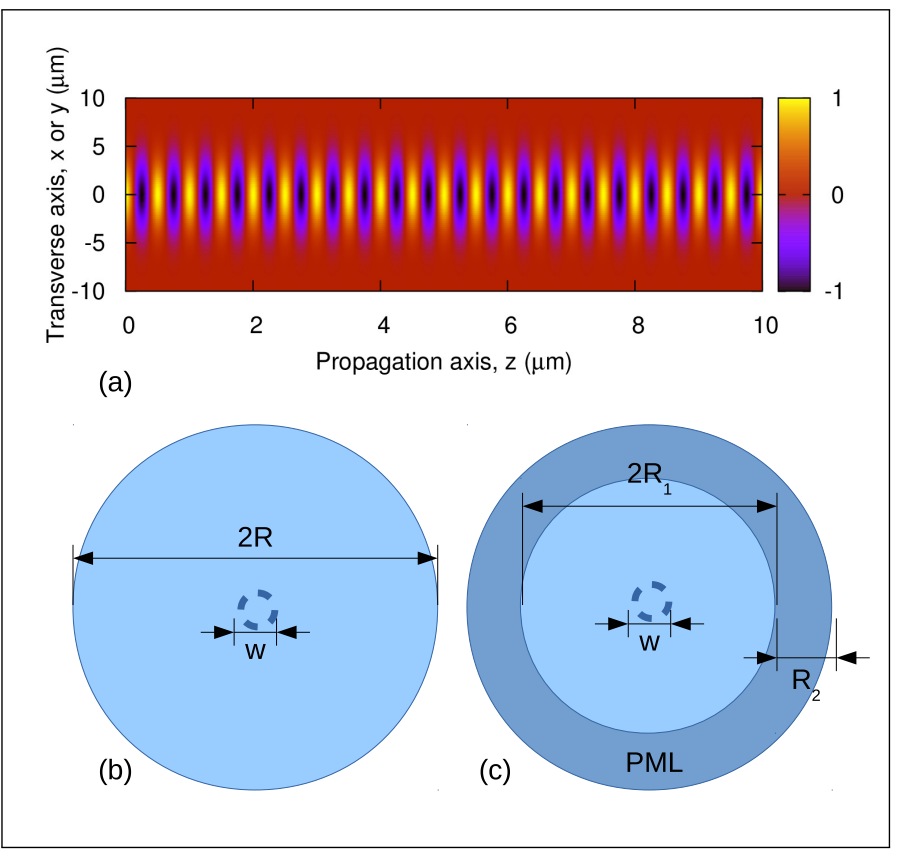

Fig. 1. Schematic representation of the model of photoelastic generation of hypersound in a homogeneous dielectric medium. (a) Two counterpropagating, detuned optical Gaussian beams interfere and create a moving spatial stress distribution. In the plot, the waist is $w=5 \mu \mathrm{m}$ and the period of the interference pattern is $0.5 \mu \mathrm{m}$. (b) Simple 2D disk-shaped domain in the transverse plane. (c) 2D disk-shaped domain terminated by a perfectly matched layer (PML) approximating an open medium.

\section{ELECTROSTRICTION MODEL}

We consider a homogeneous dielectric, solid medium. For simplicity, the medium is considered to be optically isotropic though anisotropic for elastic waves. In the Results Section, we consider either silica or silicon. The magnetic fields of the two optical beams are linearly polarized and have a transverse Gaussian dependence, according to

$$
\mathbf{H}_{i}(x, y, z ; t)=H_{0} \mathbf{h}_{i} \exp \left(-\left(x^{2}+y^{2}\right) / w^{2}\right) \exp \left(j\left(\omega_{i} t-k_{i} z\right)\right)
$$

with $i=1,2, H_{0}$ the magnetic field magnitude, $\mathbf{h}_{i}$ a transverse unit vector, and $w$ the beam waist. $\omega_{i}$ and $k_{i}$ are the angular frequency and wavevector, with $\left|k_{i}\right|=n \omega_{i} / c$ and $c$ the vacuum velocity of light. The power transported by the Gaussian beam is in the paraxial approximation

$$
P_{0}=\frac{\pi w^{2}}{4 n \varepsilon_{0} c} H_{0}^{2} .
$$

The two optical beams are counterpropagating and have a relatively small adjustable detuning $\omega=\omega_{1}-\omega_{2}$. Hence the difference of their wavenumbers $q=k_{1}-k_{2} \approx 2 k_{1}$. The square of the optical field thus contains a term proportional to $\exp (j(\omega t-q z))$ that can be phase-matched with an elastic wave providing $q \approx \omega / V$ with $V$ the phase velocity of the elastic wave. The relevant term in the electrostriction stress tensor is $T_{k l}^{e s}=-\frac{1}{2} \varepsilon_{0} p_{i j k l} D_{i}^{(1)} D_{j}^{(2) *}$ with $\mathbf{D}$ the electric displacement vector and $p_{i j k l}$ the photoelastic tensor. Note that all indices of tensors run from 1 to 3 and that we use the notation $\left(x_{1}, x_{2}, x_{3}\right)=$ $(x, y, z)$. The electric displacement vector is easily obtained for the magnetic field vector by $\frac{\partial \mathbf{D}}{\partial t}=\nabla \times \mathbf{H}$. Figure 1(a) illustrates the interference of the two optical Gaussian beams.

Given the particular form of the driving electrostriction stress, the displacement of the forced elastic wave can be assumed to be of the form [16]

$$
u_{i}(x, y, z ; t)=\hat{u}_{i}(x, y) \exp (j(\omega t-q z))
$$

This equation looks similar to a guided mode but actually describes a different situation: the driving stress oscillates in time and space along a preferred direction and the forced solution follows those oscillations while adapting its shape in the transverse plane to satisfy the appropriate boundary conditions.

We obtain the displacements $\hat{u}_{i}(x, y)$ by solving the elastodynamic equation using finite element analysis. The weak form of the equation is [5]

$$
-\omega^{2} \int_{\Omega} \rho \hat{v}_{i}^{*} \hat{u}_{i}+\int_{\Omega} S(\hat{v})_{I}^{*} c_{I J} S(\hat{u})_{J}=\int_{\Omega} S(\hat{v})_{I}^{*} T_{J}^{\mathrm{es}}
$$

with $c_{i j k l}$ the elastic tensor and $\rho$ the mass density. $\Omega$ is the domain on which $\hat{u}_{i}(x, y)$ is defined, $\mathbf{v}$ is a set if test functions satisfying the decomposition of Eq. (3), $I=(i j)$ and $J=(k l)$ are contracted indices for pairs of symmetric indices running from 1 to 6 . The strains are defined as

$$
\begin{aligned}
& S_{1}(\hat{\mathbf{u}})=\frac{\partial \hat{u}_{1}}{\partial x_{1}}, \\
& S_{2}(\hat{\mathbf{u}})=\frac{\partial \hat{u}_{2}}{\partial x_{2}}, \\
& S_{3}(\hat{\mathbf{u}})=-j q \hat{u}_{3}, \\
& S_{4}(\hat{\mathbf{u}})=\frac{\partial \hat{u}_{3}}{\partial x_{2}}-j q \hat{u}_{2}, \\
& S_{5}(\hat{\mathbf{u}})=\frac{\partial \hat{u}_{3}}{\partial x_{1}}-j q \hat{u}_{1}, \\
& S_{6}(\hat{\mathbf{u}})=\frac{\partial \hat{u}_{1}}{\partial x_{2}}+\frac{\partial \hat{u}_{2}}{\partial x_{1}} .
\end{aligned}
$$

If the domain considered is closed by an external boundary $\sigma$, the previous equations can be solved by imposing boundary conditions. In the following we will consider the domain depicted in Fig. 1(b), terminated by a free boundary condition. In order to represent a piece of homogeneous material, the solution should then decay spatially fast enough not to reach the external boundary, or otherwise reflections will occur. Of course this condition is not for granted and the absence of reflections in the solution must be checked carefully. In order to approach better the case of an infinite space, for which the actual solution should satisfy radiation conditions at infinity, one can use a perfectly matched layer (PML) [17, 18]. As depicted in Fig. 1(c), the PML surrounds the useful computation domain and is intended to absorb outgoing waves without reflecting them. PML for timeharmonic wave equations [19] can be defined as a coordinate transform from a complex infinite domain to the real finite domain [20]. The PML implementation we use is summarized in the appendix. Note that the PML may fail for waveguide-type problems in elastic media [21], and hence also in the case of our optoacoustic model. For this reason, we use a PML in the following for the case of longitudinal waves, but we have to revert to the free computation in the case of shear waves, due to numerical instabilities. 


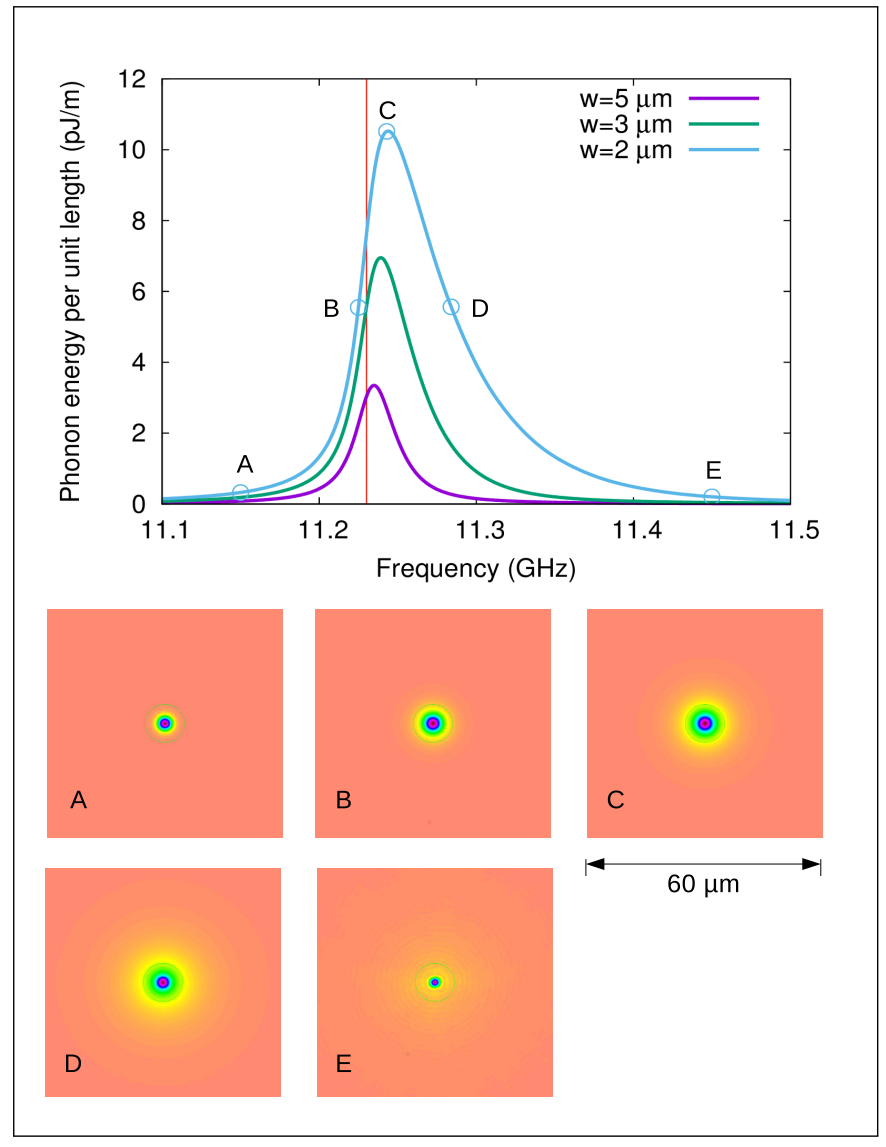

Fig. 2. optoacoustic generation of longitudinal elastic waves in silica. The two optical beams are co-polarized. The phonon energy per unit length is plotted as a function of optical detuning, for 3 different optical beam waists. Representative acoustic beam cross-sections (A-E) are shown.

\section{RESULTS}

All computations are performed for a vacuum optical wavelength of $1.55 \mu \mathrm{m}$, for silica and for silicon. There is a noticeable optoacoustic response only when the phase matching conditions of a bulk elastic wave are met. We first present results for longitudinal waves and then for shear waves. For the free model (shear waves and all acoustic beam cross-sections), we set $R=100$ $\mu \mathrm{m}$. For the PML model (longitudinal waves), we set $R_{1}=10$ $\mu \mathrm{m}$ and $R_{2}=5 \mu \mathrm{m}$.

\section{A. Longitudinal elastic waves}

We first consider silica $(n=1.458)$. Material constants are taken from Ref. [22]. Figure 2 shows the computed optoacoustic spectrum for co-polarized optical beams, for 3 different waists, as obtained with the PML model. Note that the power of the optical beams is normalized to $1 \mathrm{~W}$ in all cases. As a result, the intensity (in $\mathrm{W} / \mathrm{m}^{2}$ ) is larger for smaller waists, enhancing the optoacoustic response. The phonon energy per unit length is computed from the solution as

$$
\frac{1}{2} \omega^{2} \int_{\Omega} \rho \hat{u}_{i}^{*} \hat{u}_{i}+\frac{1}{2} \int_{\Omega} S(\hat{u})_{I}^{*} c_{I J} S(\hat{u})_{J} \cdot
$$

The longitudinal velocity for silica is $5969 \mathrm{~m} / \mathrm{s}$, so that the Brillouin frequency in this case is $11.23 \mathrm{GHz}$, as indicated by the red
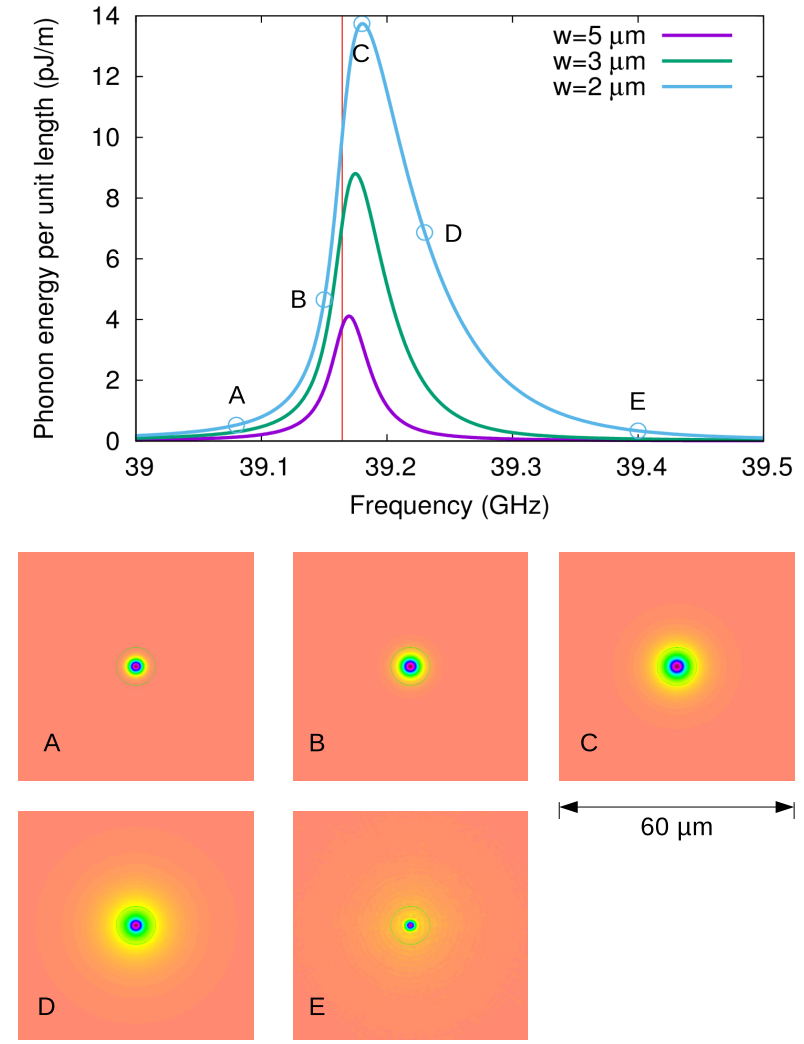

$60 \mu \mathrm{m}$

Fig. 3. optoacoustic generation of longitudinal elastic waves in silicon. The two optical beams are co-polarized. The phonon energy per unit length is plotted as a function of optical detuning, for 3 different optical beam waists. Representative acoustic beam cross-sections (A-E) are shown.

line in Figure 2. It can be seen that the response is not centered on the Brillouin frequency and is not symmetrical with respect to its maximum. This is in contrast to what is observed in the case of guided elastic modes, in optical fibers and nanoscale optical waveguides, in which case Lorentzian peaks are centered on a resonance frequency. Figure 2 further shows a selection of 5 acoustic beam cross-sections, for the $2-\mu \mathrm{m}$ waist. Away from the peak, the response is weak and strongly confined around the optical stress distribution. As the peak maximum is approached from lower frequencies, the acoustic beam width grows, but remains finitely distributed around the excitation. After the maximum, the beam width keeps on increasing and a bright center appears on top of a more extended background.

Figure 3 shows information similar to Figure 2, but for the case of silicon (with crystallographic axes aligned with the reference frame). Material constants are again taken from Ref. [22]. The refractive index $n=3.6$ and the longitudinal velocity is 8431 $\mathrm{m} / \mathrm{s}$, so that the Brillouin frequency in this case is $39.16 \mathrm{GHz}$. Apart from the change in frequency range, the characteristics of the optoacoustic response are very similar to the case of silica.

\section{B. Shear elastic waves}

In the case of shear elastic waves, a stronger optoacoustic response is obtained for cross-polarized optical beams compared to co-polarized beams. As a consequence we present results for 


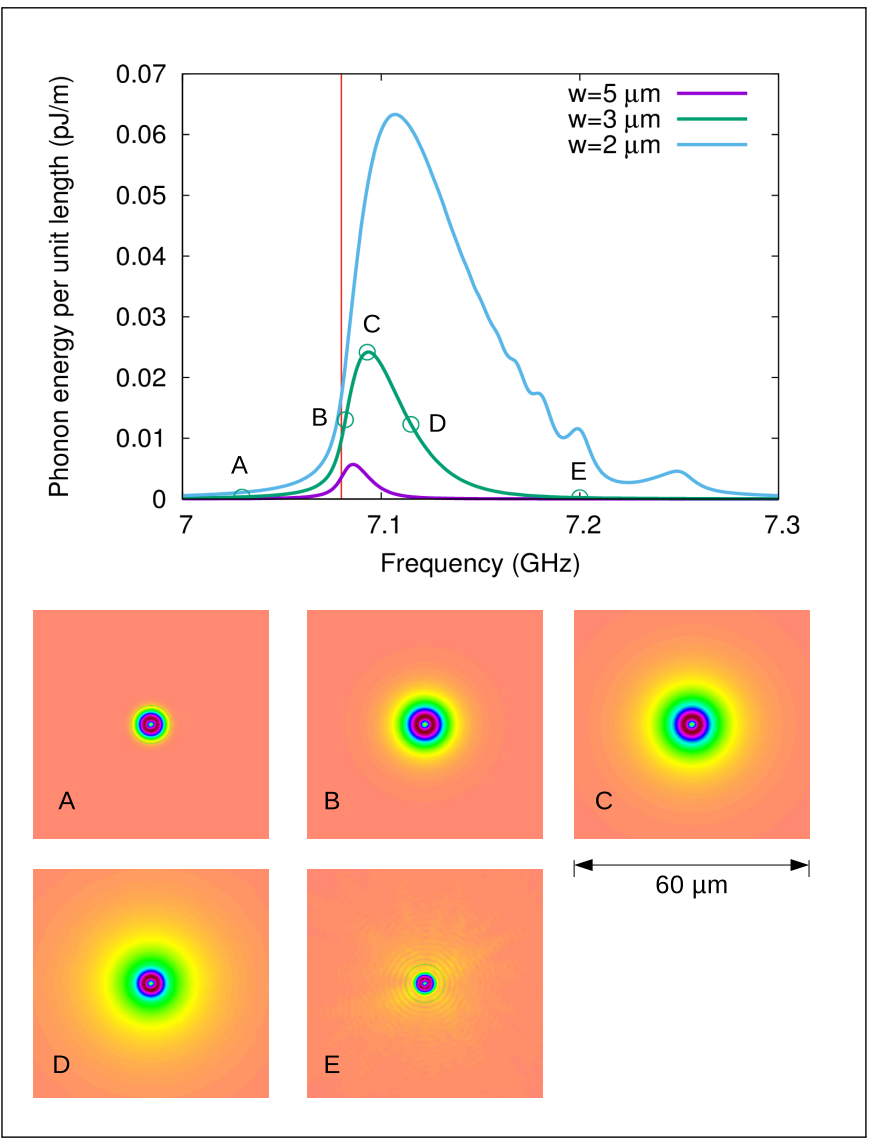

Fig. 4. optoacoustic generation of shear elastic waves in silica. The two optical beams are cross-polarized. The phonon energy per unit length is plotted as a function of optical detuning, for 3 different optical beam waists. Representative acoustic beam cross-sections (A-E) are shown.

cross-polarized beams. The shear velocity in silica is $3763 \mathrm{~m} / \mathrm{s}$, so that the Brillouin frequency in this case is $7.08 \mathrm{GHz}$. It can be seen in Figure 4 that the optoacoustic response is again not centered on the Brillouin frequency and is not symmetrical with respect to its maximum. The enhancement of the response with smaller waists is even more important. Actually, as the beam waist becomes very large, i.e. as it tends to a plane wave, the response tends toward zero. As a result, it can be said that the response is only due to the finiteness of the optical beams. For the 2- $\mu \mathrm{m}$ waist, undulations are observed on the high frequency side of the response; these are caused by reflections on the external boundary of the computation domain and indicate that the beam size has exceeded a radius of $100 \mu \mathrm{m}$. Figure 4 further shows a selection of 5 acoustic beam cross-sections, for the 3$\mu \mathrm{m}$ waist. The same phenomenon of increasing beam width is observed as in the case of longitudinal waves, but the increase is even more pronounced.

The case of shear waves in silicon, in Figure 5, shows novel features. The shear velocity is $5844 \mathrm{~m} / \mathrm{s}$, so that the Brillouin frequency in this case is $27.15 \mathrm{GHz}$. For the smaller beam waist, $5 \mu \mathrm{m}$, the response is almost centered on the Brillouin frequency and symmetrical. However, as the beam waist becomes smaller, the response becomes wider and two maxima appear before and after the Brillouin frequency. The 5 selected acoustic beam crosssections, for the $2-\mu \mathrm{m}$ waist, indicate that the acoustic beam

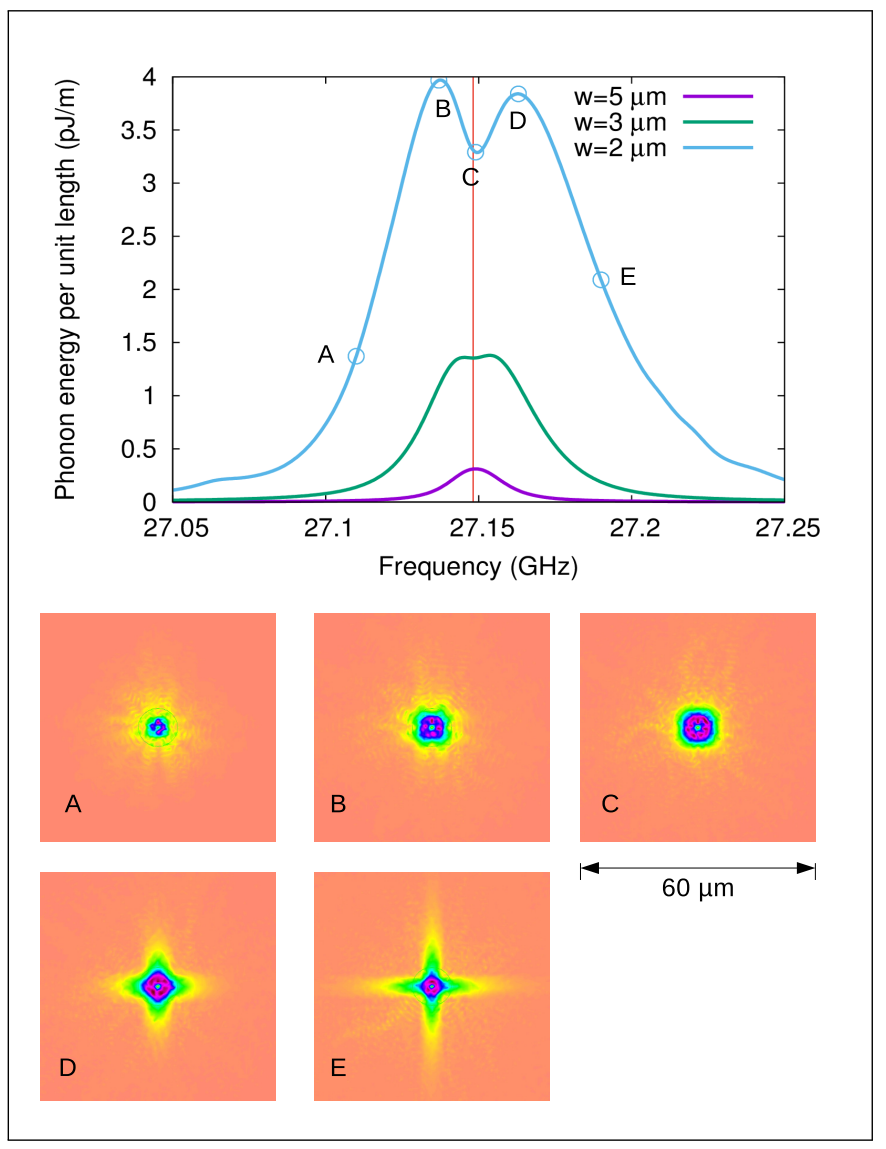

Fig. 5. optoacoustic generation of shear elastic waves in silicon. The two optical beams are cross-polarized. The phonon energy per unit length is plotted as a function of optical detuning, for 3 different optical beam waists. Representative acoustic beam cross-sections (A-E) are shown.

shape strongly depends on the anisotropy of the elastic tensor of silicon (cubic symmetry) and varies rapidly with frequency.

\section{DISCUSSION}

The most remarkable feature of the optoacoustic responses is that the generated acoustic beams remain confined around the optical stress distribution. We emphasize that there is no structural guidance, as would be provided if some region of space (a core) had a lower elastic wave velocity compared to the surrounding medium (a cladding). Instead, the finite acoustic beams are composed of a continuous superposition of the bulk elastic waves of the homogeneous medium. The Gaussian distribution of the optical beams, that translates into a Gaussian distribution of the electrostriction stress, plays an important role in this respect. Indeed, if we consider a Fourier transform in the transverse direction, the plane wave spectrum of the excitation is also a Gaussian function of the lateral wavenumber. Hence, the distribution of wavevectors is continuously distributed around its center, $(0,0, q)$, and decays rapidly away from it.

The solution of the elastodynamic Eq. (4) can be written formally as $\mathbf{u}=R(\omega) T^{\mathrm{es}}$, with $R(\omega)$ the resolvant operator. The resolvant is well-behaved, except when a mode is present in the spectrum. The bulk elastic modes of homogeneous media form slowness surfaces $[23,24]$, which are surface of revolu- 


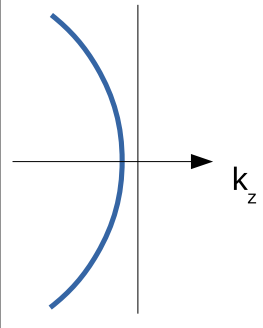

q

(a) $\omega<\omega$

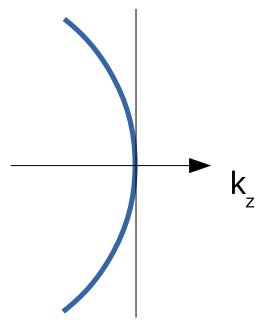

q

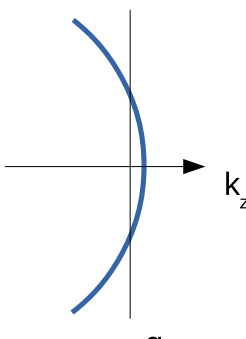

q (b) $\omega=\omega_{B}$

(c) $\omega>\omega_{B}$

Fig. 6. Intersections of the slowness surface of a bulk elastic wave with the phase-matching condition $k_{z}=q$. (a-c) Three cases ares shown depending on the magnitude of detuning frequency $\omega$ with respect to the Brillouin frequency $\omega_{B}$.

tion in wavevector space, as depicted in Figure 6. Optoacoustic phase-matching imposes $k_{z}=q$, but otherwise leaves the value of $\left(k_{x}, k_{y}\right)$ free. For a detuning frequency smaller than the Brillouin frequency, $\omega<\omega_{B}$, there is no intersection of the slowness surface with the $\left(k_{x}, k_{y}, q\right)$ plane and no bulk elastic wave can be resonantly excited. For $\omega=\omega_{B}$, there is a single osculating intersection point, whose contribution is negligible. For $\omega>\omega_{B}$, the intersection of the slowness surface with the $\left(k_{x}, k_{y}, q\right)$ plane defines a continuous curve describing the possible leakage of elastic energy to bulk waves, away from the excitation center. This leakage explains the asymmetry of the optoacoustic response: a smaller optical beam waist implies a larger plane wave spectrum and hence the excitation of bulk waves slightly away from the Brillouin frequency.

The model we have presented assumes very long optical Gaussian beams that retain the same waist along the propagation axis. This assumption is only approximate, as it is know that Gaussian beams have smoothly varying waist; taken this variation into consideration would require an extended 3D model. An alternative is the use of non-diffracting beams that can retain a constant width over rather long distances [25].

\section{CONCLUSION}

We have described a model for the generation of coherent beams of hypersound in a homogeneous solid that result from the interference of two oppositely propagating, detuned, optical Gaussian laser beams. Numerical simulations for silica and silicon predict the generation of confined hypersound beams, at frequencies close to the Brillouin frequency. The computed optoacoustic spectra are non-symmetrical, i.e. they do not affect the usual Lorentzian shape that is usually observed when modes are excited. We have explained their appearance as resulting from the excitation of a continuum of bulk elastic waves forming a slowness surface in wavevector-space. The acoustic beam widths were found to vary with detuning frequency and optical beam waist. In particular, a stronger response is found for small waists, for a constant optical power.

Agence Nationale de la Recherche (ANR) (ANR-14-CE360005-01, ANR-11-LABX-0001-01); Région de Franche-Comté (Mobilités entrantes 2016).

\section{REFERENCES}

1. R. Y. Chiao, C. H. Townes, and B. P. Stoicheff, "Stimulated Brillouin scattering and coherent generation of intense hypersonic waves," Phys. Rev. Lett. 12, 592-595 (1964).

2. L. Brillouin, "Diffusion de la lumière et des rayons $X$ par un corps transparent homogène. Influence de l'agitation thermique," Ann. Phys. (Paris) 17, 21 (1922).

3. J. Xu and R. Stroud, Acousto-optic devices: principles, design, and applications (Wiley, New York, 1992).

4. Y. R. Shen and N. Bloembergen, "Theory of stimulated Brillouin and Raman scattering," Phys. Rev. 137, A 1787 (1965).

5. V. Laude and J.-C. Beugnot, "Lagrangian description of Brillouin scattering and electrostriction in nanoscale optical waveguides," New Journal of Physics 17, 125003 (2015).

6. J. E. Sipe and M. J. Steel, "A Hamiltonian treatment of stimulated Brillouin scattering in nanoscale integrated waveguides," New Journal of Physics 18, 045004 (2016).

7. P. Dainese, P. Russell, N. Joly, J. Knight, G. Wiederhecker, H. Fragnito, V. Laude, and A. Khelif, "Stimulated Brillouin scattering from multi-GHzguided acoustic phonons in nanostructured photonic crystal fibres," Nat. Phys. 2, 388-392 (2006).

8. P. T. Rakich, C. Reinke, R. Camacho, P. Davids, and Z. Wang, "Giant enhancement of stimulated brillouin scattering in the subwavelength limit," Phys. Rev. X 2, 011008 (2012).

9. H. Shin, W. Qiu, R. Jarecki, J. A. Cox, R. H. Olsson III, A. Starbuck, Z. Wang, and P. T. Rakich, "Tailorable stimulated brillouin scattering in nanoscale silicon waveguides," Nat. Commun. 4, 1944 (2013).

10. R. Van Laer, B. Kuyken, D. Van Thourhout, and R. Baets, "Interaction between light and highly confined hypersound in a silicon photonic nanowire," Nat. Photon. 9, 199-203 (2015).

11. J.-C. Beugnot, S. Lebrun, G. Pauliat, H. Maillotte, V. Laude, and T. Sylvestre, "Brillouin light scattering from surface acoustic waves in a subwavelength-diameter optical fibre," Nat. Commun. 5, 5242 (2014).

12. B. J. Eggleton, C. G. Poulton, and R. Pant, "Inducing and harnessing stimulated brillouin scattering in photonic integrated circuits," Advances in Optics and Photonics 5, 536-587 (2013).

13. M. Merklein, B. Stiller, K. Vu, S. J. Madden, and B. J. Eggleton, "A chip-integrated coherent photonic-phononic memory," Nature Communications 8, 574 (2017).

14. P. J. Thomas, N. L. Rowell, H. M. van Driel, and G. I. Stegeman, "Normal acoustic modes and brillouin scattering in single-mode optical fibers," Phys. Rev. B 19, 4986-4998 (1979).

15. C. G. Poulton, R. Pant, and B. J. Eggleton, "Acoustic confinement and stimulated brillouin scattering in integrated optical waveguides," JOSA B 30, 2657-2664 (2013).

16. J.-C. Beugnot and V. Laude, "Electrostriction and guidance of acoustic phonons in optical fibers," Phys. Rev. B 86, 224304 (2012).

17. J.-P. Berenger, "A perfectly matched layer for the absorption of electromagnetic waves," J. Comput. Phys. 114, 185 (1994).

18. E. Bécache, S. Fauqueux, and P. Joly, "Stability of perfectly matched layers, group velocities and anisotropic waves," Journal of Computational Physics 188, 399-433 (2003).

19. I. Harari, M. Slavutin, and E. Turkel, "Analytical and numerical studies of a finite element PML for the Helmholtz equation," J. Comp. Acoust. 8, 121-137 (2000).

20. Z. S. Sacks, D. M. Kingsland, R. Lee, and J.-F. Lee, "A perfectly matched anisotropic absorber for use as an absorbing boundary condition," IEEE transactions on Antennas and Propagation 43, 1460-1463 (1995).

21. E. A. Skelton, S. D. Adams, and R. V. Craster, "Guided elastic waves and perfectly matched layers," Wave motion 44, 573-592 (2007).

22. V. Laude and J.-C. Beugnot, "Generation of phonons from electrostriction in small-core optical waveguides," AIP Advances 3, 042109042109 (2013).

23. B. A. Auld, Acoustic Fields and Waves in Solids (Wiley, New-York, 1973).

24. D. Royer and E. Dieulesaint, Elastic waves in solids (Wiley, New York, 
1999).

25. J. Durnin, "Exact solutions for nondiffracting beams. I. The scalar theory," JOSA A 4, 651-654 (1987).

\section{A. RADIAL PERFECTLY MATCHED LAYER}

The perfectly matched layer (PML) is introduced to transform an infinite, or open, problem into a finite problem. The idea is to seek a solution to the dynamical equations by using a coordinate transform from a complex infinite space, that admits evanescent waves as eigenfunctions instead of plane waves, to the real finite space. If there are only evanescent waves and they have sufficiently decayed, then the additional boundary condition terminating the PML becomes less important; a Dirichlet boundary condition is usually enforced.

Given coordinates $x$ of real space, we introduce coordinates $\boldsymbol{y}$ of complex space via a transform $y_{i}=y_{i}(\boldsymbol{x})$. Introduce the Jacobian matrix

$$
J_{i j}=\frac{\partial y_{i}}{\partial x_{j}} .
$$

In an integral, the integration element (volume) changes proportionally to $\operatorname{det}(J)$. Consider a function $u(x)=\tilde{u}(\boldsymbol{y})$. Then the gradient transforms as

$$
\nabla \tilde{u}=\frac{\partial \tilde{u}}{\partial y_{i}}=\frac{\partial x_{j}}{\partial y_{i}} \frac{\partial u}{\partial x_{j}}=J^{-t} \nabla u
$$

The inverse Jacobian has elements $J_{i j}^{-1}=\frac{\partial x_{i}}{\partial y_{j}}$. Note the transpose operator when transforming the gradient, i.e. $J_{i j}^{-t}=\frac{\partial x_{j}}{\partial y_{i}}$.

In the case of the elastodynamic equation, the weak form becomes

$$
\begin{aligned}
-\omega^{2} \int_{\Omega} \rho \hat{v}_{i}^{*} \hat{u}_{i} \operatorname{det}(J) & +\int_{\Omega} S(\hat{v})_{I}^{*} c_{I J} S(\hat{u})_{J} \operatorname{det}(J) \\
& =\int_{\Omega} S(\hat{v})_{I}^{*} T_{J}^{\mathrm{es}}
\end{aligned}
$$

with the modified definition of the strains

$$
\begin{aligned}
& s_{1}=J_{m, 1}^{-1} u_{1, m} \\
& s_{2}=J_{m, 2}^{-1} u_{2, m} \\
& S_{3}=J_{m, 3}^{-1} u_{3, m} \\
& s_{4}=J_{m, 2}^{-1} u_{3, m}+J_{m, 3}^{-1} u_{2, m} \\
& S_{5}=J_{m, 1}^{-1} u_{3, m}+J_{m, 3}^{-1} u_{1, m} \\
& S_{6}=J_{m, 1}^{-1} u_{2, m}+J_{m, 2}^{-1} u_{1, m}
\end{aligned}
$$

Let us suppose we want to attenuate waves in an angular sector, as depicted in Fig. 1(c). The PML is entered at the circular boundary satisfying $r=\sqrt{x^{2}+y^{2}}=R_{1}$. We consider a sequence of three coordinate transforms: Cartesian to polar coordinates, complexification of the radial coordinate, polar back to Cartesian coordinates. The complex polar transform is given by

$$
r^{\prime}=r+\frac{j}{\omega} \int_{R_{1}}^{r} \sigma(s) \mathrm{d} s
$$

with $\sigma(s)=\beta V\left|s-R_{1}\right| / R_{2}^{2}$, where $\beta$ is a numerical coefficient whose value can be tuned to optimize absorption. Performing the three transforms, we obtain

$$
\begin{aligned}
\operatorname{det}(J) & =1+\frac{j}{\omega} \sigma(r)=\alpha^{-1} \\
J^{-t} & =\left(\begin{array}{cc}
\left(\alpha x^{2}+y^{2}\right) / r^{2} & (\alpha-1) x y / r^{2} \\
(\alpha-1) x y / r^{2} & \left(\alpha y^{2}+x^{2}\right) / r^{2}
\end{array}\right) .
\end{aligned}
$$

